\title{
A 94GHz Differential Power Amplifier in 45nm LP CMOS
}

\author{
Noël Deferm ${ }^{1}$, Juan F. Osorio ${ }^{2}$, Anton de Graauw ${ }^{2}$ and Patrick Reynaert ${ }^{1}$
}

\author{
${ }^{1}$ K.U.Leuven ESAT-MICAS, Kasteelpark Arenberg 10, 3001 Leuven, Belgium ; \\ ${ }^{2}$ NXP Semiconductors, High Tech Campus 60, 5656 AG Eindhoven, The Netherlands
}

\begin{abstract}
This paper presents a $94 \mathrm{GHz} 4$-stage differential transformer-coupled power amplifier with capacitive neutralization. The use of transformers results in excellent common mode isolation between the different stages while providing a good impedance match. The neutralized differential pairs guarantee differential stability. The PA was designed in a $45 \mathrm{~nm}$ LP CMOS technology. An output $1 \mathrm{~dB}$ compression point of $+4 \mathrm{dBm}$ and a gain of $18 \mathrm{~dB}$ was measured. The total chip area is $0.43 \mathrm{~mm}^{2}$ and the active part consumes only $0.07 \mathrm{~mm}^{2}$. The $3 \mathrm{~dB}$ bandwidth is $14 \mathrm{GHz}$. Power consumption is $120 \mathrm{~mW}$ from a $1 \mathrm{~V}$ supply, resulting in a peak PAE of $4.6 \%$.
\end{abstract}

Index Terms - mm-wave, CMOS, power amplifier, differential design, $\mathrm{W}$-band.

\section{INTRODUCTION}

CMOS technology scaling has pushed the operating frequency of RF circuits towards the mm-wave region. In particular, the band around $94 \mathrm{GHz}$ has its purpose for imaging applications in the medical and security/surveillance sector. CMOS can greatly reduce the production cost for these applications thanks to its high integration capabilities. To design CMOS circuits at these high frequencies, traditional mm-wave design methods have to be reinvestigated and adapted. Indeed, single ended W-band design methods based on transmission lines often result in a large chip area. Furthermore, the large capacitive feedback through the gate-drain capacitance limits the high-frequency performance of MOS transistors. The presented 4-stage differential transformer-coupled common source PA with capacitive neutralization solves these problems. The use of transformers and slow wave differential transmission lines results in the lowest active area reported to date. Capacitive neutralization cancels the internal feedback which leads to the highest reported gain and very good stability performance at $94 \mathrm{GHz}$.
In section II the design techniques that are adopted in the PA are explained. Section III focuses on the stability behaviour of the PA. In section IV the measurement results and the comparison with the simulations are discussed.

\section{POWER AMPLIFIER DESIGN AND TOPOLOGY}

Figure 1 shows the schematic of the PA. Compared to a single-ended topology, a differential design has better rejection against substrate noise coming from digital circuits. Furthermore, a differential design also results in large benefits for the performance of the mm-wave circuit. First of all, due to the close placement of the differential transistors, a very good virtual ground is achieved at the source of these devices. Source degeneration, which can be a problem in single ended designs, is thus minimized.

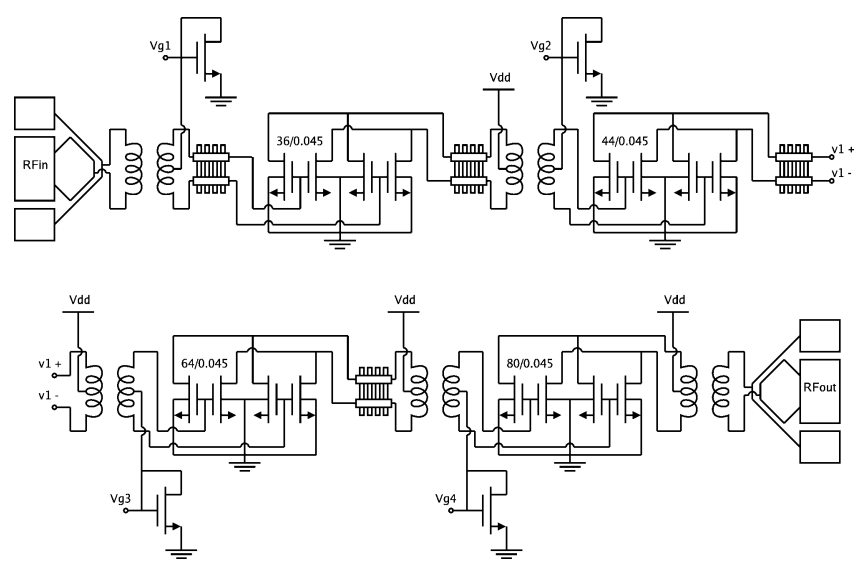

Fig. 1. Circuit schematic of the $94 \mathrm{GHz}$ PA.

Secondly, the differential design approach also offers new impedance matching network topologies. Integrated differential transformers, which have excellent performance even at these high frequencies [4], consume a very small area, offer an elegant solution for biasing and supply voltage connection at the center tap and can perform an impedance match for transforming the input 
impedance of a certain stage to the wanted load impedance for the previous stage. The combination of slow-wave differential transmission lines with these transformers creates low-area robust matching networks with very good common mode isolation and precise transformed impedance values. The transformers used in this design use stacked metal layers and have diameters starting from $20 \mu \mathrm{m}$ up to $45 \mu \mathrm{m}$.

The transformers were designed together with the slow wave transmission lines in such a way that the load impedance of the transistors are as close as possible to the optimal point for maximum output power and still achieve good gain performance. These loads were optimized with a combination of a load pull simulation together with a gain analysis for each transistor stage, starting from the output differential pair up to the input pair. Figure 2 shows the trade off between load optimization for output power and gain of the output stage of the PA.

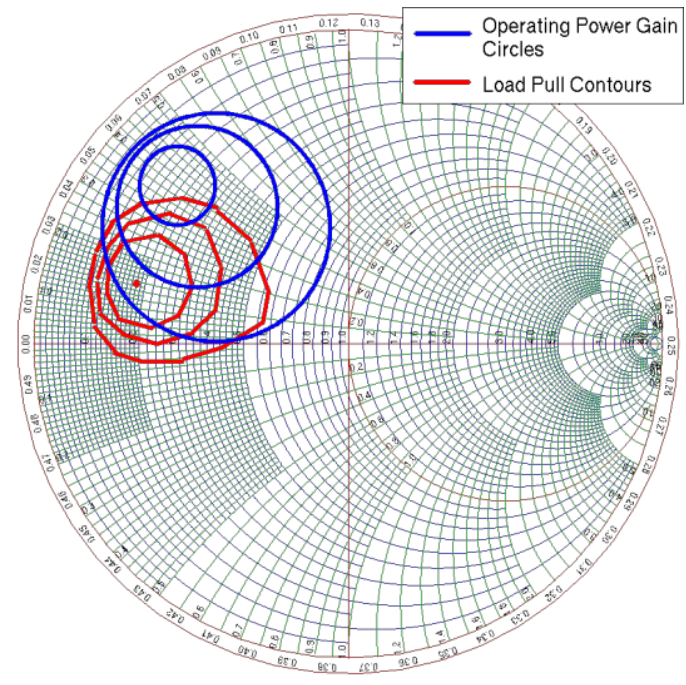

Fig. 2. Simulated load pull contours and operating power gain circles.

\section{AMPLIFIER STABILITY ANALYSIS}

Stability is another major design issue at mm-wave frequencies, especially in CMOS due to the high parasitic gate-drain capacitance. A $45 \mathrm{~nm}$ transistor with a finger width of $1 \mu \mathrm{m}$ is not unconditionally stable at $94 \mathrm{GHz}$. This means that both gain and stability are compromised by internal feedback through the gate-drain capacitance of the device. This effect limits the maximum gain that can be achieved from one stage, thus resulting in a higher DC power consumption as more stages need to be cascaded. The differential topology offers an elegant design solution to simultaneously achieve high gain and stability. Indeed, the transistor can be pushed into the unconditionally stable region without losing gain, by placing cross-coupled capacitors in parallel with the unwanted gate-drain capacitor [6] [7]. This neutralizes the internal feedback factor and allows achieving higher gain for the same DC power consumption. In this amplifier, capacitive neutralization was achieved by placing a cross-coupled differential transistor pair in parallel with the amplifying transistor pair (see figure 1). The area overhead of this approach is very small compared to other neutralization techniques. Furthermore, this technique is more robust to process variations since the amplifying and neutralizing transistors are matched.

This capacitive neutralization technique results in an excellent stability performance. The necessary and sufficient conditions for the amplifier to be unconditionally stable are [5]:

$$
\mathrm{K}>1
$$

And

$$
|\Delta|<1
$$

Figure 4 shows the measured $\mathrm{k}$-factor and the absolute value of $\Delta$. The lowest $\mathrm{K}$ value is about 4 at $90 \mathrm{GHz}$ which is well above the stability limit. The highest absolute value of $\Delta$ is 0.1 at $102 \mathrm{GHz}$. This means that the power amplifier is in the unconditionally stable range.

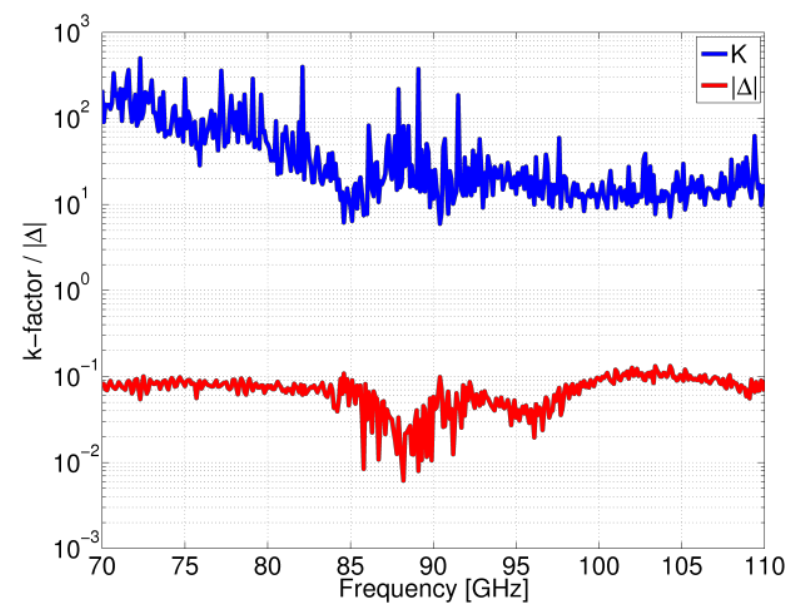

Fig. 3. Measured K and delta of the PA

Common mode stability is another point of concern. Indeed, at lower frequencies, the transistors have more intrinsic gain which can lead to common-mode instability. To improve the common-mode stability, a high impedance common mode path between the different stages was implemented. This high-impedance common mode path is inherently present by using transformers between the stages. 


\section{MEASUREMENT RESULTS}

Figure 4 shows the large signal measurements and simulation results at $91 \mathrm{GHz}$. As can be seen, accurate modeling of all parasitics results in a close match between simulation and measurement results. The output $1 \mathrm{~dB}$ compressed output power is $+4 \mathrm{dBm}$. The maximum measured output power is $+7.6 \mathrm{dBm}$. Due to measurement setup limitations the PA could not be pushed into complete saturation. According to simulations the saturated output power would be around $+8 \mathrm{dBm}$.

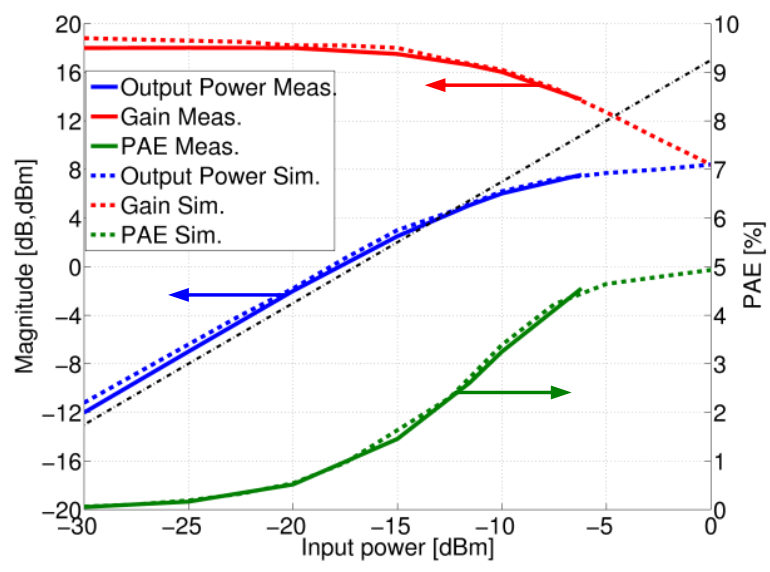

Fig. 4. Measured and simulated output power, gain and PAE.

With a power consumption of $120 \mathrm{~mW}$ at a supply voltage of $1 \mathrm{~V}$ a measured peak PAE of $4.6 \%$ is achieved. Output power measurements were also performed from 87 up to $97 \mathrm{GHz}$ (Figure 5). Within this band, the $1 \mathrm{~dB}$ compression point of the PA is always higher than $+3 \mathrm{dBm}$. All these large signal measurements were done with a calibrated power meter.

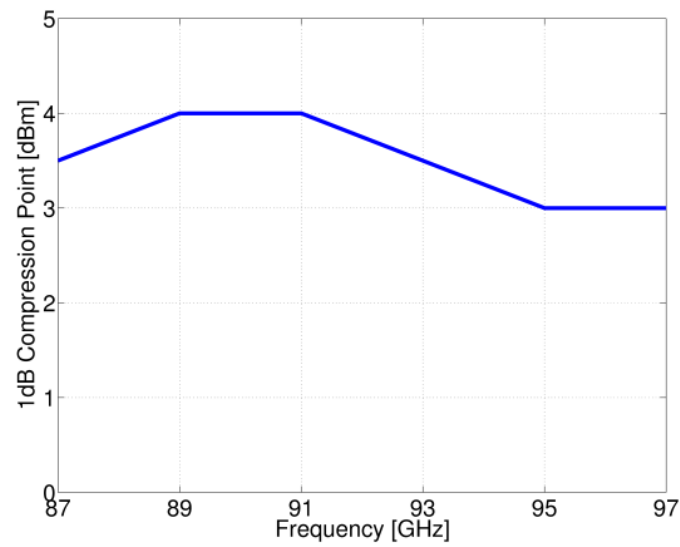

Fig. 5. Measured $\mathrm{CP} 1 \mathrm{~dB}$ from $87 \mathrm{GHz}$ up to $97 \mathrm{GHz}$.
The small signal measurements and simulation results are shown in figure 6 . The measured gain peaked at $90 \mathrm{GHz}$ with a value of $18.5 \mathrm{~dB}$. This is the highest gain ever reported for a $\mathrm{W}$-band CMOS power amplifier.

The input return loss was lower than $-12 \mathrm{~dB}$ from 82.5 up to $96.5 \mathrm{GHz}$ with a minimum value between -30 and $40 \mathrm{~dB}$ at $89 \mathrm{GHz}$. Despite the fact that the output matching network was optimized for maximum output power and not for maximum gain like the input matching network, a measured output return loss of $-9 \mathrm{~dB}$ for the complete band was still achieved.

The measurements fit the simulations very good for S21 and S22. The difference between the simulated and measured S11 can be explained due to the fact that the input return loss is very sensitive to gate impedance variations which are very hard to model correctly. The impact of this error on the gain is very small due to the fact that the overall input return loss is very low in the band of operation.

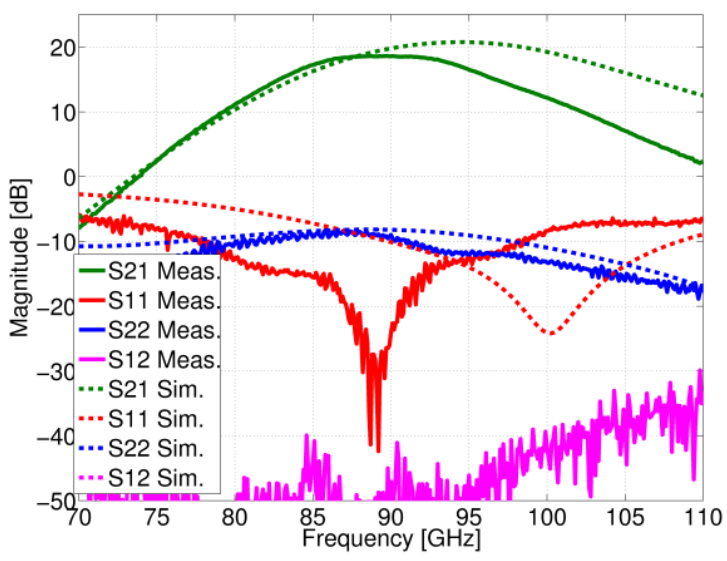

Fig. 6. Measured and simulated s-parameters.

The total chip area is $0.43 \mathrm{~mm}^{2}$. This includes probepads, bond-pads, ESD-protection and decouple capacitors. The actual PA only consumes $0.07 \mathrm{~mm}^{2}$ which is denoted as the active area on figure 7 . This very low area consumption is achieved thanks to the use of compact transformers and short slow-wave differential transmission lines. To our knowledge, this is the smallest active area reported for a W-band CMOS amplifier. Figure 7 shows the photograph of the PA.

Compared to other state of the art W-band CMOS power amplifiers, this PA achieves the highest gain at the lowest supply voltage, the smallest active area and the best ITRS figure of merit for PA's (ITRS PA FoM $=f^{2} x$ Gain x PAE x Psat) [8]. Table I shows the comparison with other PA's. 


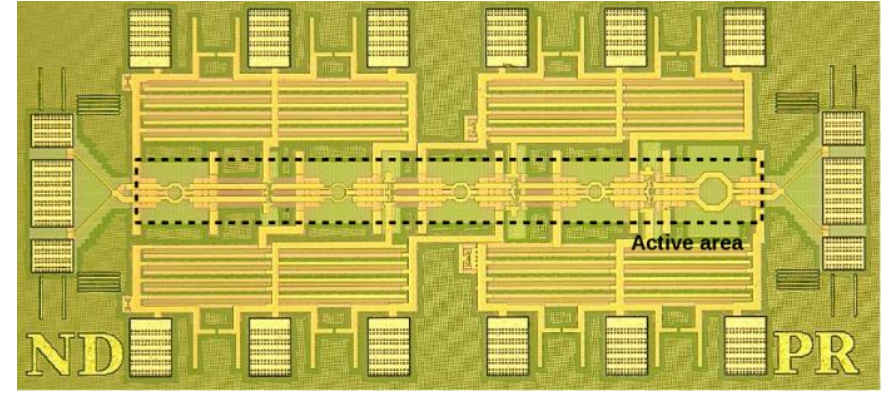

Fig. 7. Chip photograph.

TABLE I

COMPARISON WITH OTHER W-BAND CMOS PA'S

\begin{tabular}{|c|c|c|c|c|}
\hline Reference & {$[1]$} & {$[2]$} & {$[3]$} & This work \\
\hline Technology & $\begin{array}{c}90 \mathrm{~nm} \text { RF } \\
\text { CMOS }\end{array}$ & $\begin{array}{c}\text { 90nm RF } \\
\text { CMOS }\end{array}$ & $\begin{array}{c}65 \mathrm{~nm} \\
\text { CMOS }\end{array}$ & $\begin{array}{c}\mathbf{4 5 n m} \\
\text { LP CMOS }\end{array}$ \\
\hline Freq. [GHz] & 96 & 100 & 100 & $\mathbf{9 4}$ \\
\hline Topology & $\begin{array}{c}3 \text { st. } \\
\text { casc. SE }\end{array}$ & $\begin{array}{c}3 \text { st. casc. } \\
\text { (1 st. SE, } \\
\text { 2 st. } \\
\text { Balanced) }\end{array}$ & $\begin{array}{c}\text { SE } \\
\text { St. CS } \\
\text { differentia } \\
\mathbf{1}\end{array}$ \\
\hline Supply [V] & 2.5 & 2.4 & 1.2 & $\mathbf{1}$ \\
\hline Gain [dB] & 16 & 15 & 13 & $\mathbf{1 8 . 5}$ \\
\hline $\begin{array}{c}3 \mathrm{~dB} \mathrm{BW} \\
{[\mathrm{GHz}]}\end{array}$ & 22 & 17 & 21 & $\mathbf{1 4}$ \\
\hline OCP [dBm] & +2 & +6 & +6 & $+\mathbf{4}$ \\
\hline Psat [dBm] & +4 & +10 & +10 & $+\mathbf{7 . 6}$ \\
\hline PDC [mW] & 54 & 160 & 86 & $\mathbf{1 2 0}$ \\
\hline $\begin{array}{c}\text { Total area } \\
{\left[\mathrm{mm}^{2}\right]}\end{array}$ & 0.42 & 0.40 & 0.32 & $\mathbf{0 . 4 3}$ \\
\hline $\begin{array}{c}\text { Active area } \\
{\left[\mathrm{mm}^{2}\right]}\end{array}$ & 0.28 & 0.24 & 0.19 & $\mathbf{0 . 0 7}$ \\
\hline Peak PAE [\%] & 4.1 & 5.2 & 7.3 & $\mathbf{4 . 6}$ \\
\hline $\begin{array}{c}\text { ITRS FOM } \\
\text { [W.GHz }\end{array}$ & $\mathbf{3 8}$ & $\mathbf{1 6 4}$ & $\mathbf{1 4 6}$ & $\mathbf{1 6 6}$ \\
\hline
\end{tabular}

\section{CONCLUSION}

A CMOS W-band differential power amplifier was presented in this paper. The PA achieves an output $1 \mathrm{~dB}$ compression point of $+4 \mathrm{dBm}$ and a gain above $18 \mathrm{~dB}$ which is the highest reported for W-band CMOS PA's. The ITRS Figure-Of-Merit for power amplifiers [7] for this work is $166 \mathrm{~W} . \mathrm{GHz}^{2}$ which is also the highest reported. The $3 \mathrm{~dB}$ bandwidth ranges from $82.5 \mathrm{GHz}$ up to $96.5 \mathrm{GHz}$. Power consumption is $120 \mathrm{~mW}$ at a supply voltage of $1 \mathrm{~V}$. A measured peak PAE of $4.6 \%$ is achieved.
With an active area of only $0.07 \mathrm{~mm}^{2}$ this is the smallest $94 \mathrm{GHz}$ PA reported to date. Furthermore, the power consumption is low despite the differential nature of the circuit. This is due to the high gain that is achieved per stage, resulting from the capacitive neutralization and the low loss transformers.

\section{ACKNOWLEDGEMENT}

The authors would like to thank IWT Flanders for the financial support, and Raf Roovers from NXP research Eindhoven for the many discussions. TELEMIC and Rhode and Schwarz are also acknowledged for measurement support.

\section{REFERENCES}

[1] Y.-S. Jiang, Z.-M. Tsai, J.-H. Tsai, H.-T. Chen, and H. Wang, "A 86-to-108 GHz Amplifier in 90nm CMOS," IEEE Microwave and Wireless Components Letters, vol. 18, no. 2, pp. 124-126, Feb. 2008.

[2] Y.-S. Jiang, J.-H. Tsai, and H. Wang, "A W-Band Medium Power Amplifier in 90nm CMOS," IEEE Microwave and Wireless Components Letters, vol. 18, no. 12, pp. 818-820, Dec. 2008.

[3] D. Sandström, M. Varonen, M. Kärkkäinen, and K. Halonen, "W-Band CMOS Amplifiers Achieving $+10 \mathrm{dBm}$ Saturated Output Power and 7.5dB NF," ISSCC Dig. Tech. Papers, pp. 486-487, Feb. 2009.

[4] D. Chowdhury, P. Reynaert, A. M. Niknejad, "A $60 \mathrm{GHz} 1 \mathrm{~V}+12.3 \mathrm{dBm}$ Transformer-Coupled Wideband PA in 90nm CMOS," ISSCC Dig. Tech. Papers, pp. 560635, Feb. 2008.

[5] G. Gonzalez, "Microwave Transistor Amplifiers: Analysis and Design, $2^{\text {nd }}$ edition," Prentice-Hall, Inc, ISBN 0-13-254335-4, 1996.

[6] N. Deferm, P. Reynaert, "A $100 \mathrm{GHz}$ TransformerCoupled Fully Differential Amplifier in $90 \mathrm{~nm}$ CMOS," IEEE RFIC 2010 Symp. Dig., pp. 359-362, May 2010.

[7] W.L. Chan, J.R. Long, "A 58-65 GHz Neutralized CMOS Power Amplifier with PAE Above 10\% at 1-V Supply," Solid-State Circuits, IEEE Journal of, vol. 45, no. 3, pp. 554-564, March 2010.

[8] R. Brederlow, W. Weber, S. Donnay, P. Wambacq, J. Sauerer, M. Vertergt, "A Mixed-Signal Design Roadmap," IEEE Digest and Test of Computers," vol. 18, issue 6, pp. 34-46, Nov./Dec. 2001. 